\title{
A SIMPLE PROOF OF AN IDENTITY OF RAMANUJAN
}

\section{D. HIRSCHHORN}

(Received 11 May 1981; revised 24 December 1981)

Communicated by A. J. van der Poorten

\begin{abstract}
One of Ramanujan's unpublished, unproven identities has excited considerable interest over the years. Indeed, no fewer than four proofs have appeared in the literature. The object of this note is to present yet another proof, simpler than the others, relying only on Jacobi's triple product identity.
\end{abstract}

1980 Mathematics subject classification (Amer. Math. Soc.): 10 A 45.

The identity referred to in the title is

$$
\prod_{n \geqslant 1} \frac{\left(1-q^{n}\right)^{5}}{\left(1-q^{5 n}\right)}=1-5 \sum_{n \geqslant 1} \frac{\chi(n) n q^{n}}{1-q^{n}}
$$

where

$$
\chi(n)=\left\{\begin{aligned}
+1 & \text { if } n \equiv \pm 1(\bmod 5) \\
-1 & \text { if } n \equiv \pm 2(\bmod 5) \\
0 & \text { if } n \equiv 0(\bmod 5)
\end{aligned}\right.
$$

Ramanujan stated this identity in an unpublished manuscript, and proofs have been given by Darling (1921), Mordell (1922) and Bailey (1952a, b). Andrews (1980) also discussed the identity.

C Copyright Australian Mathematical Society 1983 
My object is to present a simple proof of (1.1), depending only on Jacobi's triple product identity

$$
\prod_{n \geqslant 1}\left(1+a q^{2 n-1}\right)\left(1+a^{-1} q^{2 n-1}\right)\left(1-q^{2 n}\right)=\sum_{-\infty}^{\infty} a^{n} q^{n^{2}},
$$

a proof of which may be found in Hirschhorn (1976).

We start by proving the identity

$$
\begin{aligned}
\prod_{n \geqslant 1}\left(1+a q^{2 n-1}\right. & )\left(1+a^{-1} q^{2 n-1}\right)\left(1+b q^{2 n-1}\right)\left(1+b^{-1} q^{2 n-1}\right)\left(1-q^{2 n}\right)^{2} \\
= & \left\{\prod_{n \geqslant 1}\left(1+a b^{2} q^{10 n-5}\right)\left(1+a^{-1} b^{-2} q^{10 n-5}\right)\right. \\
& \times\left(1+a^{2} b^{-1} q^{10 n-5}\right)\left(1+a^{-2} b q^{10 n-5}\right) \\
& +a q \prod_{n \geqslant 1}\left(1+a b^{2} q^{10 n-3}\right)\left(1+a^{-1} b^{-2} q^{10 n-7}\right) \\
& \times\left(1+a^{2} b^{-1} q^{10 n-1}\right)\left(1+a^{-2} b q^{10 n-9}\right) \\
& +a^{-1} q \prod_{n \geqslant 1}\left(1+a b^{2} q^{10 n-7}\right)\left(1+a^{-1} b^{-2} q^{10 n-3}\right) \\
& \times\left(1+a^{2} b^{-1} q^{10 n-9}\right)\left(1+a^{-2} b q^{10 n-1}\right) \\
& +b q \prod_{n \geqslant 1}\left(1+a b^{2} q^{10 n-1}\right)\left(1+a^{-1} b^{-2} q^{10 n-9}\right) \\
& \times\left(1+a^{2} b^{-1} q^{10 n-7}\right)\left(1+a^{-2} b q^{10 n-3}\right) \\
& +b^{-1} q \prod_{n \geqslant 1}\left(1+a b^{2} q^{10 n-9}\right)\left(1+a^{-1} b^{-2} q^{10 n-1}\right) \\
& \left.\quad \times\left(1+a^{2} b^{-1} q^{10 n-3}\right)\left(1+a^{-2} b q^{10 n-7}\right)\right\} \\
& \times \prod_{n \geqslant 1}\left(1-q^{10 n}\right)^{2}
\end{aligned}
$$

We have by (1.2),

$$
\begin{gathered}
\prod_{n \geqslant 1}\left(1+a q^{2 n-1}\right)\left(1+a^{-1} q^{2 n-1}\right)\left(1+b q^{2 n-1}\right)\left(1+b^{-1} q^{2 n-1}\right)\left(1-q^{2 n}\right)^{2} \\
=\sum_{r, s=-\infty}^{\infty} a^{r} b^{s} q^{r^{2}+s^{2}},
\end{gathered}
$$


which we choose to write

$$
=\sum_{n=-\infty}^{\infty} \sum_{r+2 s=n} a^{r} b^{s} q^{r^{2}+s^{2}} .
$$

We now consider the five cases $n=5 m, n=5 m+1, n=5 m-1, n=5 m+2$, $n=5 m-2$.

In the first, set $r=m+2 t, \quad s=2 m-t$,

in the second, $\quad r=m+1+2 t, \quad s=2 m-t$, in the third, $\quad r=m-1+2 t, \quad s=2 m-t$, in the fourth, $\quad r=m+2 t, \quad s=2 m+1-t$, and in the last, $r=m+2 t, \quad s=2 m-1-t$, and the sum becomes

$$
\begin{aligned}
\sum_{-\infty}^{\infty} a^{m} b^{2 m} q^{5 m^{2}} \cdot \sum_{-\infty}^{\infty} a^{2 t} b^{-t} q^{5 t^{2}} & +a q \sum_{-\infty}^{\infty} a^{m} b^{2 m} q^{5 m^{2}+2 m} \cdot \sum_{-\infty}^{\infty} a^{2 t} b^{-t} q^{5 t^{2}+4 t} \\
& +a^{-1} q \sum_{-\infty}^{\infty} a^{m} b^{2 m} q^{5 m^{2}-2 m} \cdot \sum_{-\infty}^{\infty} a^{2 t} b^{-t} q^{5 t^{2}-4 t} \\
& +b q \sum_{-\infty}^{\infty} a^{m} b^{2 m} q^{5 m^{2}+4 m} \cdot \sum_{-\infty}^{\infty} a^{2 t} b^{-t} q^{5 t^{2}-2 t} \\
& +b^{-1} q \sum_{-\infty}^{\infty} a^{m} b^{2 m} q^{5 m^{2}-4 m} \cdot \sum_{-\infty}^{\infty} a^{2 t} b^{-t} q^{5 t^{2}+2 t},
\end{aligned}
$$

which, again by (1.2), yields (2.1).

We now prove (1.1). In (2.1), put $-a^{2} q$ for $a,-a^{4} q$ for $b$, then $q$ for $q^{2}$, multiply by $a^{3}$, make use of (1.2), and we obtain

$$
\begin{aligned}
& \left(a^{3}-a-a^{-1}+a^{-3}\right) \\
& \times \prod_{n \geqslant 1}\left(1-a^{2} q^{n}\right)\left(1-a^{-2} q^{n}\right)\left(1-a^{4} q^{n}\right)\left(1-a^{-4} q^{n}\right)\left(1-q^{n}\right)^{2} \\
& =\prod_{n \geqslant 1}\left(1-q^{5 n-3}\right)\left(1-q^{5 n-2}\right)\left(1-q^{5 n}\right) \\
& \quad \times \sum_{-\infty}^{\infty}(-1)^{n}\left(a^{10 n-3}+a^{-10 n+3}\right) q^{\left(5 n^{2}-3 n\right) / 2} \\
& -\prod_{n \geqslant 1}\left(1-q^{5 n-4}\right)\left(1-q^{5 n-1}\right)\left(1-q^{5 n}\right) \\
& \quad \times \sum_{-\infty}^{\infty}(-1)^{n}\left(a^{10 n-1}+a^{-10 n+1}\right) q^{\left(5 n^{2}-n\right) / 2}
\end{aligned}
$$


If we now differentiate with respect to $a$, multiply by $a$, differentiate with respect to $a$ again and set $a=1$, we obtain

$$
\begin{aligned}
& 16 \prod_{n \geqslant 1}\left(1-q^{n}\right)^{6} \\
& =\prod_{n \geqslant 1}\left(1-q^{5 n-3}\right)\left(1-q^{5 n-2}\right)\left(1-q^{5 n}\right) \sum_{-\infty}^{\infty}(-1)^{n} 2(10 n-3)^{2} q^{\left(5 n^{2}-3 n\right) / 2} \\
& -\prod_{n \geqslant 1}\left(1-q^{5 n-4}\right)\left(1-q^{5 n-1}\right)\left(1-q^{5 n}\right) \sum_{-\infty}^{\infty}(-1)^{n} 2(10 n-1)^{2} q^{\left(5 n^{2}-n\right) / 2} \\
& =\prod_{n \geqslant 1}\left(1-q^{5 n-3}\right)\left(1-q^{5 n-2}\right)\left(1-q^{5 n}\right)\left(18+80 q \frac{d}{d q}\right) \sum_{-\infty}^{\infty}(-1)^{n} q^{\left(5 n^{2}-3 n\right) / 2} \\
& -\prod_{n \geqslant 1}\left(1-q^{5 n-4}\right)\left(1-q^{5 n-1}\right)\left(1-q^{5 n}\right)\left(2+80 q \frac{d}{d q}\right) \sum_{-\infty}^{\infty}(-1)^{n} q^{\left(5 n^{2}-n\right) / 2} \\
& =\prod_{n \geqslant 1}\left(1-q^{5 n-3}\right)\left(1-q^{5 n-2}\right)\left(1-q^{5 n}\right) \\
& \times\left(18+80 q \frac{d}{d q}\right) \prod_{n \geqslant 1}\left(1-q^{5 n-4}\right)\left(1-q^{5 n-1}\right)\left(1-q^{5 n}\right) \\
& -\prod_{n \geqslant 1}\left(1-q^{5 n-4}\right)\left(1-q^{5 n-1}\right)\left(1-q^{5 n}\right) \\
& \times\left(2+80 q \frac{d}{d q}\right) \prod_{n \geqslant 1}\left(1-q^{5 n-3}\right)\left(1-q^{5 n-2}\right)\left(1-q^{5 n}\right) \\
& =\prod_{n \geqslant 1}\left(1-q^{5 n-3}\right)\left(1-q^{5 n-2}\right)\left(1-q^{5 n}\right) \\
& \times \prod_{n \geqslant 1}\left(1-q^{5 n-4}\right)\left(1-q^{5 n-1}\right)\left(1-q^{5 n}\right) \\
& \times\left\{18-80 \sum_{n \geqslant 1}\left[\frac{(5 n-4) q^{5 n-4}}{1-q^{5 n-4}}+\frac{(5 n-1) q^{5 n-1}}{1-q^{5 n-1}}+\frac{5 n q^{5 n}}{1-q^{5 n}}\right]\right\} \\
& -\prod_{n \geq 1}\left(1-q^{5 n-4}\right)\left(1-q^{5 n-1}\right)\left(1-q^{5 n}\right) \\
& \times \prod_{n \geqslant 1}\left(1-q^{5 n-3}\right)\left(1-q^{5 n-2}\right)\left(1-q^{5 n}\right) \\
& \times\left\{2-80 \sum_{n \geqslant 1}\left[\frac{(5 n-3) q^{5 n-3}}{1-q^{5 n-3}}+\frac{(5 n-2) q^{5 n-2}}{1-q^{5 n-2}}+\frac{5 n q^{5 n}}{1-q^{5 n}}\right]\right\} \\
& =\prod_{n \geqslant 1}\left(1-q^{5 n-4}\right)\left(1-q^{5 n-3}\right)\left(1-q^{5 n-2}\right)\left(1-q^{5 n-1}\right)\left(1-q^{5 n}\right)^{2}
\end{aligned}
$$




$$
\begin{aligned}
\times\left\{16-80 \sum_{n \geqslant 1}\left[\frac{(5 n-4) q^{5 n-4}}{1-q^{5 n-4}}-\frac{(5 n-3) q^{5 n-3}}{1-q^{5 n-3}}\right.\right. \\
\left.\left.-\frac{(5 n-2) q^{5 n-2}}{1-q^{5 n-2}}+\frac{(5 n-1) q^{5 n-1}}{1-q^{5 n-1}}\right]\right\} \\
=\prod_{n \geqslant 1}\left(1-q^{n}\right)\left(1-q^{5 n}\right) \times\left\{16-80 \sum_{n \geqslant 1} \frac{\chi(n) n q^{n}}{1-q^{n}}\right\},
\end{aligned}
$$

as required.

\section{References}

G. E. Andrews (1980) 'Ramanujan's “lost” notebook III: The Rogers-Ramanujan continued fraction,' Dept. of Mathematics Research Report, The Pennsylvania State University.

W. N. Bailey (1952a) 'A note on two of Ramanujan's formulae,' Quart. J. Math. Oxford Ser. (2) 3, $29-31$

W. N. Bailey (1952b) 'A further note on two of Ramanujan's formulae,' Quart J. Math. Oxford Ser. (2) $3,158-160$.

H. B. C. Darling (1921) 'Proofs of certain identities and congruences enunciated by S. Ramanujan,' Proc. London Math. Soc. (2) 19, 350-372.

M. D. Hirschhorn (1976) 'Simple proofs of identities of MacMahon and Jacobi,' Discrete Math. 16, $161-162$.

L. J. Mordell (1922) "Note on certain modular relations considered by Messrs Ramanujan, Darling and Rogers,' Proc. London Math. Soc. (2) 20, 408-416.

\section{School of Mathematics}

University of New South Wales

Kensington, N.S.W. 2033

Australia 